\title{
Narrativa
}

\section{Dissonanze e incontri possibili: il dibattito sul nuovo realismo nella letteratura contemporanea in Italia, Francia e Germania}

\section{Christian Rivoletti}

\section{(2) OpenEdition Journals \\ Edizione digitale \\ URL: https://journals.openedition.org/narrativa/881 \\ DOI: $10.4000 /$ narrativa.881 \\ ISSN: 2804-1224 \\ Editore}

Presses universitaires de Paris Nanterre

\section{Edizione cartacea}

Data di pubblicazione: 1 décembre 2016

Paginazione: 173-183

ISBN: 978-2-84016-266-7

ISSN: $1166-3243$

Notizia bibliografica digitale

Christian Rivoletti, «Dissonanze e incontri possibili: il dibattito sul nuovo realismo nella letteratura contemporanea in Italia, Francia e Germania», Narrativa [Online], 38 | 2016, online dal 01 janvier 2022, consultato il 22 février 2022. URL: http://journals.openedition.org/narrativa/881 ; DOI: https://doi.org/ 10.4000/narrativa.881

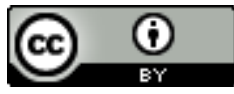

Narrativa est mise à disposition selon les termes de la Licence Creative Commons Attribution 4.0 International. 


\section{Dissonanze e incontri possibili: il dibattito sul nuovo realismo nella letteratura contemporanea in Italia, Francia e Germania}

U no dei momenti salienti del dibattito critico sulla letteratura contemporanea è rappresentato dall'indagine e dalla discussione, avviatesi negli ultimi anni e tuttora in corso, dedicate al fenomeno del "nuovo realismo". Anche se non sono affatto mancate occasioni di scambio a livello internazionale, il dibattito su questo fenomeno si è sinora sviluppato sostanzialmente all'interno delle singole aree nazionali. Il presente contributo intende avviare un confronto e una riflessione a cavallo di tre dei maggiori ambiti europei (Italia, Francia e Germania), segnalando, accanto a analogie e divergenze, anche possibili interazioni e sinergie tra le diverse modalità di approccio a questo fenomeno ${ }^{1}$.

A tal fine, dopo una sintetica ricognizione del dibattito italiano $(\$ 1)$, si analizzerà in quale misura quel dibattito sia stato recepito in Francia $(\$ 2)$ e in Germania $(\mathbb{3} 3)$. Contestualmente mi sono anche chiesto quanto la ricezione del dibattito straniero abbia interagito (in ognuno dei due casi) con la percezione del fenomeno all'interno della singola letteratura nazionale, e ne sia stata eventualmente influenzata. Dal confronto emergono alcune conclusioni e prospettive di lavoro.

\section{TRE NODI DEL DIBATTITO ITALIANO}

Due eventi, pressoché contemporanei (entrambi risalgono al 2008), vengono solitamente ricordati come punto di partenza del dibattito italiano sul nuovo

1. Espongo qui alcuni risultati di un più ampio progetto di ricerca di taglio comparatistico, attualmente in corso e dedicato al fenomeno del nuovo realismo in Francia, Germania e Italia. Il progetto, dal titolo "Darstellung der Wirklichkeit und Hybridisierung der Formen”, ha ottenuto un finanziamento della Deutsche Forschungsgemeinschaft. 
realismo: la relazione New Italian Epic, presentata da Wu Ming 1 nel quadro del convegno Up Close \& Personal tenutosi presso la McGill University di Montréal ${ }^{2}$, e la sezione tematica della rivista Allegoria dedicata al "ritorno alla realtà" nella letteratura e nel cinema ${ }^{3}$. Sebbene già in precedenza il problema del realismo fosse stato variamente riproposto al centro dell'attenzione $\mathrm{critica}^{4}$, fu in particolare l'indagine condotta da Allegoria a accendere un vero e proprio dibattito, con posizioni spesso contrapposte 5 .

Tre questioni mi paiono imprescindibili per riassumere la riflessione e il dibattito sul tema del nuovo realismo: provo a ricordarle perché ci serviranno da guida anche nel confronto successivo con le aree francese e tedesca. La prima riguarda la cronologia del fenomeno. Sebbene sia l'apertura del saggio New Italian Epic, sia la prima domanda di un questionario rivolto agli scrittori contemporanei nel fascicolo di Allegoria evocassero la data simbolica e traumatica dell'11 settembre 2001, esiste tuttavia un certo accordo nel fissare l'inizio del fenomeno letterario del ritorno alla realtà agli anni Novanta. Questa è, ad

2. Il convegno Up Close \& Personal. Workshop sulla narrativa italiana contemporanea (Montréal, 28-29 marzo 2008) fu organizzato da Eugenio Bolongaro e Francesco Borghesi presso il Department of Italian Studies della McGill University. La relazione di Wu Ming 1 venne pubblicata successivamente in volume: Wu Ming, New Italian Epic. Letteratura, sguardo obliquo ritorno al futuro, Torino, Einaudi, 2009.

3. Donnarumma, Raffaele, Policastro, Gilda, Taviani, Giovanna (a cura di), Ritorno alla realtà? Narrativa e cinema alla fine del postmoderno, Allegoria, n. 57, 2008, pp. 7-93.

4. Mi limito a ricordare Bertoni, Federico, Realismo e letteratura. Una storia possibile, Torino, Einaudi, 2007; CASADEI, Alberto, Stile e tradizione nel romanzo italiano contemporaneo, Bologna, Il Mulino, 2007.

5. Si vedano la "controinchiesta" di Cortellessa, Andrea, Lo stato delle cose (con interventi di scrittori e di critici), in Specchio + (supplemento settimanale della Stampa) del novembre 2008 (consultabile anche in https://issuu.com/passi.falsi/docs/cortellessa) e il dibattito che ne seguì sul sito Nazione Indiana (https:/ /www.nazioneindiana. com). Sulla vicenda rimando a GANERI, Margherita, "Reazioni allergiche al concetto di realtà. Il dibattito intorno al numero 57 di «Allegoria»", in SERKowSKA Hanna (a cura di), Finzione Cronaca Realtà. Scambi, intrecci e prospettive nella narrativa italiana contemporanea, Massa, Transeuropa, 2011, pp. 51-68. Tra i numerosi interventi che in Italia hanno affrontato la questione del "ritorno alla realtà" e del nuovo realismo, ricordo inoltre i seguenti volumi: POLESE, Ranieri (a cura di), Il romanzo della politica, la politica nel romanzo, Parma, Guanda, 2008 (in part. si veda il contributo dello stesso Polese, "Ritorno al reale. In principio fu il noir", pp. 7-13); SpInAzzola, Vittorio (a cura di), Tirature '10. Il New Italian Realism, Milano, Il Saggiatore, 2010; Giglioli, Daniele, Senza trauma. Scrittura dell'estremo e narrativa del nuovo millennio, Macerata, Quodlibet, 2011; FERRARIS, Maurizio, Manifesto del nuovo realismo, Roma-Bari, Laterza, 2012; DE CARo, Mario, Ferraris, Maurizio (a cura di), Bentornata realtà. Il nuovo realismo in discussione, Torino, Einaudi, 2012; Di Cesare, Donatella, Ocone, Corrado, Regazzoni, Simone (a cura di), Il nuovo realismo è un populismo, Genova, Il Melangolo, 2013 e SiTI, Walter, Il realismo è l'impossibile, Roma, Nottetempo, 2013. 
esempio, la posizione di Raffaele Donnarumma, che fa coincidere tale svolta con i segni di un esaurimento del postmodernismo in letteratura ${ }^{6}$. Nella stessa direzione vanno anche le osservazioni di Raffaello Palumbo Mosca, che registra una "torsione verso il mondo [...] a partire dai primi anni Novanta" e che menziona in particolare quattro opere: Occhio per Occhio. La pena di morte in quattro storie e Cronache italiane (entrambe del 1992) di Sandro Veronesi, Fattacci (1997) di Vincenzo Cerami e Maggio selvaggio (1999) di Edoardo Albinati. A queste seguono poi Antonio Franchini, L'abusivo (2001) e Roberto Saviano, Gomorra (2006), che va dunque considerato come "la punta dell'iceberg" di un fenomeno ben più esteso 7 . Riguardo alla cronologia, si dovrà infine osservare che il libro di Saviano, che è stato indicato da più parti come il simbolo di questa svolta realistica - sebbene non ne rappresenti certamente (come credo anche io) l'inizio, ma piuttosto il momento più vistoso -, ha in qualche modo sancito questa svolta, dando avvio in Italia (con ritardo rispetto alla Francia, come vedremo meglio più avanti) al dibattito critico.

Il riferimento a Gomorra ci consente di toccare inoltre una seconda questione di rilievo relativa al fenomeno del realismo contemporaneo: quella del rapporto tra forme e contenuto. Al dibattito sul nuovo realismo si collega infatti (non soltanto in Italia, ma anche all'estero, come vedremo) l'antico pregiudizio che l'attenzione al realismo possa equivalere a una disattenzione o, ancor peggio, a una svalutazione, da parte di scrittori e di critici, del lavoro artistico sulle forme ${ }^{8}$. Nella fattispecie, il rischio che consegue a questa posizione è quello di non considerare la ricerca di forme e di strutture narrative particolari, una ricerca che invece caratterizza il nuovo realismo contemporaneo, pervenendo spesso a costruzioni formali ibride, come la commistione tra genere romanzesco, scrittura saggistica o documentaria, narrazione autobiografica, reportage giornalistico e altre forme di scrittura. A mio avviso, questa elaborazione e ibridazione

6. Cfr., già nel citato fascicolo tematico di Allegoria, l'ampio contributo di DoNNARUMma, Raffaele, "Nuovi realismi e persistenze postmoderne: narratori italiani di oggi", e poi ID., Ipermodernità. Dove va la narrativa contemporanea, Bologna, Il Mulino, 2014.

7. Palumbo Mosca, Raffaello, L'invenzione del vero. Romanzi ibridi e discorso etico nell'Italia contemporanea, Roma, Gaffi, 2014 (le citazioni sono tratte rispettivamente da pp. 22 e 12).

8. Esemplare, in proposito, perché sintomatica di un bisogno di azzerare i contenuti (e la loro capacità, un domani, di trasmettere ancora un messaggio critico) a vantaggio assoluto delle forme, la posizione assunta da Andrea Cortellessa riguardo a Gomorra di Matteo Garrone (CorTellessa, Andrea, "Reale, troppo reale", https://www.nazioneindiana.com/2008/10/29/reale-troppo-reale). 
delle forme tradizionali rappresenta un fattore peculiare del realismo contemporaneo (e molto spesso anche una chiave del suo successo"), in virtù del quale esso si differenzia dai realismi letterari del passato - un fattore che, di per sé, dovrebbe essere oltretutto sufficiente a chiarire anche quel tanto di equivoco che l'espressione "ritorno a" reca con sé: a "tornare" è infatti sì un interesse della letteratura per il mondo reale, ma ciò avviene in forme sostanzialmente nuove, che mostrano che gli autori in qualche modo "hanno fatto tesoro" dell'esperienza e della riflessione postmoderna sulle forme letterarie e sulle "difficoltà" del testo di rapportarsi alla realtà.

All'elaborazione formale si lega una terza e ultima questione che gioca un ruolo centrale per la caratterizzazione del realismo contemporaneo: la funzione dell'autore e del narratore. Anche all'interno del dibattito italiano è stato osservato che la narrativa attuale si caratterizza per una "riabilitazione del soggetto" Dopo che la teoria letteraria postmoderna aveva decretato la "morte dell'autore" "11, si assiste oggi a una sorta di paradosso, in virtù del quale proprio nell'alveo del realismo (fenomeno storicamente connesso, in misura prevalente, al concetto di oggettività), il soggetto scrivente diviene di nuovo importante. Nei testi contemporanei, esso è infatti spesso proprio il garante e il responsabile, a livello etico, di quella realtà che racconta. In un mondo dominato dai media, nel quale sperimentiamo continuamente il pericolo della contraffazione, emerge un forte bisogno di ricorrere all'esperienza concretamente vissuta, una forte rivalutazione della testimonianza diretta. Sta dunque all'autore non mettersi da parte nel tentativo di offrirci (come accadeva ad esempio nel naturalismo di fine Ottocento) una rappresentazione quanto più possibile "oggettiva" del reale; il suo compito diviene bensì quello di prendere parte alla rappresenta-

9. Sul fenomeno dell'ibridazione e in particolare sulla novità e sull'efficacia della struttura della forma ibrida adottata da Saviano in Gomorra, mi permetto di rinviare a Rivoletti, Christian, "Forma ibrida e logica poetica: il realismo in Gomorra di Roberto Saviano", in Rivoletti, Christian, Tortora, Massimiliano (a cura di), Forme del realismo nella letteratura italiana tra modernismo e contemporaneità, Allegoria, n. 71-72 (2016), pp. 98-114. Un'indagine specifica, mirata a "capire in quale misura la non-fiction ha cambiato le regole del gioco al punto da minare il tradizionale assetto dei generi nel sistema letterario", è stata condotta da RiCCIARDI, Stefania, Gli artifici della non-fiction. La messinscena narrativa in Albinati, Franchini, Veronesi, Massa, Transeuropa, 2011 (la citazione è tratta da p. 18).

10. Cfr. Donnarumma, Raffaele, Ipermodernità, cit., pp. 125-134. Al fenomeno della rivalutazione del soggetto scrivente, si lega inoltre il successo di un genere letterario quale l'autofiction.

11. Il riferimento è alla nota tesi di BARTHES, Roland, "La mort de l'auteur" (1968), in ID., Le bruissement de la langue, Paris, Éditions du Seuil, 1984, pp. 61-67. 
zione e alla narrazione che ci offre, di intromettersi e di "sporcarsi le mani", di esporsi e di farsi carico della responsabilità etica propria di chi scrive del mondo.

\section{COMPARABILITÀ E INVITO AL CONFRONTO: \\ IL DIBATTITO SUL NUOVO REALISMO IN FRANCIA}

Va ricordato che il dibattito sul nuovo realismo nella letteratura italiana, avviatosi anche grazie al già menzionato convegno di Montréal, è proseguito e si è arricchito anche tramite numerosi e significativi momenti d'incontro e di discussione realizzatisi fuori d'Italia. Tra questi vanno annoverate, oltre a varie iniziative della stessa rivista Narrativa che ospita il presente contributo, anche alcune occasioni d'incontro realizzate a Varsavia, a Toronto e, più recentemente, a Parigi, alle quali hanno preso parte italianisti attivi sia all'estero, sia in Italia ${ }^{12}$.

Se il mutuo scambio testimoniato da questi eventi basterebbe già a dimostrare la ricezione del dibattito italiano nell'area francese, è tuttavia importante considerare anche un altro aspetto, ovvero la presenza di una riflessione autonoma in Francia, già molto precoce, sul fenomeno del nuovo realismo all'interno della propria letteratura. In maniera forse più sommessa e senza suscitare un dibattito equiparabile a quello italiano, le espressioni "retour au réel" e "écrire le réel", utilizzate in relazione a autori e opere contemporanee, hanno infatti iniziato a circolare nella critica francese già a partire dal 2001 grazie alle analisi di Dominique Viart, e successivamente agli interventi di Jean Bessière ${ }^{13}$. Il fatto che in Francia esistessero già un'idea e addirittura un'espressione corrispondente ("retour au réel") a quelle poste al centro dei dibattiti italiani ha

12. Tra i contributi dedicati al rapporto tra letteratura contemporanea e realtà, vorrei menzionare almeno il fascicolo di CONTARINI, Silvia (a cura di), Letteratura e azienda. Rappresentazioni letterarie dell'economica e del lavoro nell'Italia degli anni 2000, Narrativa, n. 31-32, 2010. Gli atti del convegno Les nouveaux réalismes dans la culture italienne à l'aube du troisième millénaire. Définitions et mises en perspective (Parigi, 12-14 giugno 2014), organizzato dall'Università Sorbonne Nouvelle-Paris 3 in collaborazione con l'Università Paris Nanterre, si possono leggere adesso nel volume di Contarini, Silvia, De PaulisDalembert, Maria Pia, Tosatti, Ada (a cura di), Nuovi realismi: il caso italiano. Definizioni, questioni, prospettive, Massa, Transeuropa, 2016. Gli interventi dei convegni di Varsavia e di Toronto sono stati pubblicati rispettivamente in: SERkowska Hanna (a cura di), Finżione Cronaca Realtà, cit. e Somigl, Luca (a cura di), Negli archivi e per le strade. Il ritorno alla realtà nella narrativa di inizio millennio, Ariccia, Aracne, 2013.

13. VIART, Dominique, "Écrire au présent : l'esthétique contemporaine", in TouRET, Michèle, Dugast, Francine (a cura di), Le temps des lettres. Quelles périodisations pour l'histoire de la littérature française du XX ${ }^{\mathrm{e}}$ siècle? , Rennes, Presses universitaires de Rennes, 2001, pp. 317-336; BÉssIÈre, Jean, Qu'est-il arrivé aux écrivains français? D'Alain RobbeGrillet à Jonathan Littell, Loverval, Labor, 2006. 
indubbiamente reso più facilmente percepibile e recepibile la problematica discussa in Italia, nonostante le innegabili differenze esistenti tra le rispettive riflessioni sul fenomeno del realismo contemporaneo. Non è questa la sede per un resoconto dettagliato del dibattito critico francese (compito che attende ancora di venir assolto), ma tali differenze emergono chiaramente anche soltanto da una rapida occhiata ai tre nodi già menzionati riguardo al dibattito italiano.

Innanzitutto è diversa la cronologia del fenomeno, che in Francia viene fatto solitamente risalire agli inizi degli anni Ottanta: lo stesso Viart e Bruno Vercier datano ad esempio il momento in cui "la littérature renoue avec le réel" al 1982, anno nel quale compaiono le opere Sortie d'usine di François Bon e L'Excès l'usine di Leslie Kaplan ${ }^{14}$. Il fenomeno stesso, dunque, e non solamente la sua tematizzazione in ambito critico, si realizza con un netto anticipo rispetto all'area italiana.

Diversa è inoltre l'angolazione dalla quale si guarda al problema del rapporto tra forma e contenuto. Sintomatica è in proposito la scelta di Viart che, ricordando "l'interdit formel qui pèse sur le récit "réaliste", decide di parlare di "écritures du réel": realizza così una sorta di compromesso tra il concetto di "écriture" (che rimanda alla stagione teorica che ha dominato in Francia durante i decenni precedenti) e quello di riscoperta del reale (che contrassegna invece il presente $)^{15}$. É probabile che la lunga traversata nel mare delle teorie sostanzialmente "antirealiste" del secondo Novecento, da Alain Robbe-Grillet e il Nouveau Roman a Roland Barthes, dallo strutturalismo e post-strutturalismo sino al decostruzionismo ${ }^{16}$, sia la causa di quel tono, che ho definito più "sommesso" di quello italiano, nel denunciare un ritorno della letteratura alla realtà. Se questa è soltanto un'ipotesi, è invece un fatto che tale ritorno, partito con anticipo, perduri negli anni Duemila: basti guardare, accanto alle opere più recenti dello

14. Viart, Dominique, Vercier, Bruno, La littérature française au présent. Héritage, modernité, mutations [2005], Paris, Bordas, 2008, p. 213.

15. Ibid., p. 207. Secondo Viart, sia in Kaplan che in Bon si registra una rinuncia al racconto, rinuncia necessaria al realismo e che viene qui rifunzionalizzata alla rappresentazione efficace di una realtà (quella dell'officina) mutilata della dimensione identitaria e sociale (ibid., pp. 213-218).

16. Per uno sguardo retrospettivo sulla trafila di queste posizioni teoriche in rapporto al problema del realismo, cfr. Asholt, Wolfgang, "Un renouveau du "réalisme" dans la littérature contemporaine?", nel fascicolo tematico Problèmes du réalisme dans la littérature française contemporaine della rivista Lendemains - Etudes comparées sur la France, 38, n. 150/151, 2013, pp. 22-35 (23-26). 
stesso Bon (Daewoo, 2004), a libri come L'Adversaire (2000) di Emmanuel Carrère o Carnets de Homs (2012) di Jonathan Littell.

In particolare questi ultimi due testi, oltre a presentare sperimentazioni formali e procedure di ibridazione che sono sì di volta in volta diverse e specifiche, ma pur sempre perfettamente confrontabili con fenomeni che caratterizzano il panorama italiano (e ciò, spesso, anche grazie all'elaborazione originale di modelli comuni, tratti dalla letteratura anglofona), offrono esempi interessanti della funzione del soggetto scrivente, mettendoci in entrambi i casi di fronte a un autore che opta per un programmatico coinvolgimento diretto del proprio io a garanzia dei fatti che narra ${ }^{17}$. $\mathrm{Al}$ di là del fenomeno dell'autore che diviene personaggio-testimone (come in Carrère e in Littell), la creazione di una categoria critica delle "écritures de soi" per caratterizzare una parte ampia e significativa della produzione contemporanea conferma infine il rilievo ricoperto da questo aspetto all'interno del dibattito francese ${ }^{18}$.

Grazie anche alla sua prossimità linguistica e a una tradizionalmente forte penetrazione dell'italianistica italiana, l'ambito francese rappresenta dunque un terreno particolarmente favorevole a un confronto comparatistico con l'area italiana che, in virtù delle dinamiche sopra accennate di affinità e di "scarto", potrebbe condurre a risultati fecondi, permettendo di declinare in maniera più articolata e approfondita l'indagine sul paradigma del nuovo realismo contemporaneo e di arricchire reciprocamente il quadro critico-letterario nazionale.

\section{DiFFERENZE E MOTIVI D'INCONTRO: L'AMBITO TEDESCO}

Ben diversa dai panorami italiano e francese è invece la situazione della Germania, dove l'attenzione critica per il fenomeno del nuovo realismo all'interno della letteratura nazionale è sorta molto tardi e incontra ancora oggi resistenze teoriche, e dove le espressioni "Rückkehr zum Realismus", "Wiederkehr des Realismus" o "Zurück zur Wirklichkeit" sono state sostanzialmente importate dalle aree francese e italiana.

Sebbene esista un ampio consenso nel considerare il 1989 come momento storico di svolta nella produzione culturale e letteraria tedesca, e nonostante

17. Per un confronto tra Gomorra e L'Adversaire relativamente ai procedimenti di ibridazione delle forme e alla presenza del narratore, con un riferimento alla dialettica instaurata da entrambi i testi con il comune modello di Truman Capote, rimando a RivoletTi, Christian, "Forma ibrida e logica poetica: il realismo in Gomorra di Roberto Saviano", cit.

18. Viart, Dominique, Vercier, Bruno, La littérature française au présent, cit., pp. 29-130. 
alcune voci critiche abbiano individuato tra le conseguenze di questa svolta la nascita di una tendenza "neorealistica"19 (che, pur generatasi in seno ad avvenimenti nazionali, condividerebbe tuttavia grosso modo la cronologia dei fenomeni francesi e italiani), non sono sorti in Germania né un'indagine critica specifica, né un dibattito sul tema.

La categoria critica di "nuovo realismo" è piuttosto, a ben vedere, un prodotto d'importazione. Due autorevoli specialisti della letteratura francese, Rita Schober (esponente di spicco della filologia romanza nella Repubblica Democratica e studiosa del realismo francese otto e novecentesco) e Wolfgang Asholt, riconobbero prontamente la svolta realistica all'interno della narrativa francese, intervenendo con due contributi dal titolo programmatico (rispettivamente Renowveau du réalisme? e Rückkehr zum Realismus?) nel 200220. Allora il concetto non penetrò tuttavia nei dibattiti dei germanisti. È significativo che sia stato un convegno bilaterale italo-tedesco (Villa Vigoni, 2012) a porre espressamente la questione di una svolta dopo il postmoderno e dell'esistenza, nel panorama letterario contemporaneo tedesco, di "un'intera serie di testi, che sembrano rendere giustizia al bisogno di realtà" [Wirklichkeitsbedürfnis], segnando final-

19. Sul 1989 come data di svolta concordano le principali storie della letteratura tedesca contemporanea, da SCHNELL, Ralf, Geschichte der deutschsprachigen Literatur seit 1945, Stuttgart, Metzler, 2003 a BARNER, Wilfried, Geschichte der deutschen Literatur von 1945 bis zur Gegenwart, München, Beck, 2006. Più recentemente, PETERSDORFF, Dirk von, Literaturgeschichte der Bundesrepublik. Deutschland. Von 1945 bis zur Gegenwart, München, Beck, 2011, p. 115, ha sostenuto che "nella letteratura contemporanea [tedesca] domina un neorealismo [ein Neorealismus]", collegando tale osservazione al romanzo Faserland (1995) di Christian Kracht senza tuttavia caratterizzarla o approfondirla ulteriormente. Sul realismo in Kracht vedi anche Vogler, Ingo, "Die Ästhetisierung des Realitätsbezugs: Christian Krachts 'Ich werde hier sein im Sonnenschein und im Schatten' zwischen Realität und Fiktion”, in Krumrey Birgitta, Vogler, Ingo, Derlin, Katharina (a cura di), Realitätseffekte in der deutschsprachigen Gegenwartsliteratur. Schreibweisen nach der Postmoderne?, Heidelberg, Winter, 2014, pp. 161-178.

20. Schober, Rita, "Renouveau du réalisme? Ou de Zola à Houellebecq? Hommage à Colette Becker", in La représentation du réel dans le roman. Mélanges offerts à Colette Becker, Paris, Oséa, 2002, pp. 333-344; Asнolt, Wolfgang, "Die Rückkehr zum Realismus? Écritures du quotidien bei François Bon und Michel Houellebecq", in Gelz, Andreas, Eтте, Ottmar (a cura di), Der französischsprachige Roman heute. Theorie des Romans - Roman der Theorie in Frankereich und der Frankophonie, Tübingen, Stauffenburg, 2002, pp. 93-110. Di Schober si veda anche il successivo "Vision du monde et théorie du roman, concepts opératoires des romans de Michel Houellebecq", in Blanckeman, Bruno et alii (a cura di), Le roman francais au tournant du XXI siècle, Paris, Presses de la Sorbonne Nouvelle, 2004, pp. 505-515. Sempre connesso al panorama francese è il contributo di Link, Jürgen, “'Wiederkehr des Realismus' - aber welches? Mit besonderem Bezug auf Jonathan Littell", nel fascicolo tematico di LiNK, Jürgen, PArR, Rolph (a cura di), Neues Wissen - neuer Realismus?, KultuRRevolution, n. 54, 2008, pp. 6-23. 
mente l'avvio di un dibattito nella germanistica tedesca - dibattito condotto tuttavia con grande circospezione, come indica il prudenziale ricorso al concetto barthesiano di "effetti di realtà" 21 . Sempre dall'area romanza sono provenuti altri stimoli. In seguito all'invito del filosofo Maurizio Ferraris presso il Käte Hamburger Kolleg Recht als Kultur di Bonn e alla traduzione tedesca (2014) del suo Manifesto del nuovo realismo, il quotidiano Die Zeit ha dedicato una nutrita serie di articoli al fenomeno del realismo ${ }^{22}$. Infine, nell'ambito delle discipline romanze, sono sorti infine due convegni tenutisi presso l'Università di Costanza: Erfabrung und Referenz, di taglio comparatistico (2012) e Zurück zur Wirklichkeit. Rekurrenzen des Realismus in der italienischen Literatur des 20. und 21. Jahrbunderts, organizzato allo scopo di creare un primo momento d'incontro e di riflessione sul fenomeno del nuovo realismo tra italianisti tedeschi e italiani $(2014)^{23}$.

Come emerge dal volume Neue Realismen in der Gegenwartsliteratur (2016) che fa finalmente il punto sul fenomeno del nuovo realismo nella letteratura contemporanea tedesca ${ }^{24}$, nel dibattito critico tedesco sembra ancora forte il bisogno di

21. Il convegno Nach der Postmoderne. Formen und Funktionen des "Realitätseffekts" in der Deutschsprachigen Gegenwartsliteratur (Villa Vigoni, 31 settembre - 3 ottobre 2012) fu organizzato dai germanisti tedeschi e italiani Albert Meier, Alessandro Costazza, Dirk Niefanger, Matteo Galli; gli atti sono stati pubblicati in Krumrey Birgitta et alii (a cura di), Realitätseffekte in der deutschsprachigen Gegenwartsliteratur, cit. (la citazione è da p. 10, trad. di chi scrive). Nel titolo e in vari contributi si fa riferimento alla categoria elaborata da BARTHES, Roland, "L'effet de réel" (1968), in ID., Le bruissement de la langue, cit., pp. 167-174.

22. Ferraris, Maurizio, Manifest des neuen Realismus, Frankfurt a.M., Klostermann, 2014; cfr. inoltre le edizioni del 3, 16 e 24 aprile, 15 maggio, 5 e 18 giugno e 3 luglio 2014 del quotidiano Die Zeit. Tra i segnali di attenzione per il fenomeno del "ritorno al reale" lanciati in Germania già in precedenza, vanno tuttavia segnalate almeno le seguenti due iniziative, entrambe in ambito interdisciplinare: il fascicolo tematico di Bohrer, Karl Heinz e Scheel, Kurt (a cura di), Wirklichkeit! Wege in die Realität, Merkur, 59, 9/10, 2005, dedicato al tema dell'attuale "nostalgia della realtà" ("Sehnsucht nach Wirklichkeit"), senza riferimenti però a una svolta realistica nel panorama letterario contemporaneo) e la Scuola di Dottorato di Ricerca dell'Università di Costanza Das Reale in der Kultur der Moderne.

23. Si vedano gli atti pubblicati dagli organizzatori del convegno Rüth, Axel, Schwarze, Michael (a cura di), Erfahrung und Referen₹. Erzählte Geschichte im 20. Jahrbundert, Paderborn, Fink 2016. Una prima parte dei contributi del convegno italo-tedesco Zurück zur Wirklichkeit, organizzato Michael Schwarze, Jobst Welge e da chi scrive sono confluiti nel fascicolo tematico di Rivoletti, Christian, Tortora, Massimiliano (a cura di), Forme del realismo..., cit., mentre una seconda parte a cura di RivoletTI, Christian, Schwarze, Michael, Welge, Jobst è in corso di stampa in Germania per la rivista on line Romanische Studien.

24. Fauth, Søren R., PArR, Rolf (a cura di), Neue Realismen in der Gegenwartsliteratur, München, Fink, 2016 (per una rassegna degli interventi critici sul nuovo realismo in ambito tedesco, rimando in particolare alle pp. 11-14). 
fare i conti con le categorie teoriche del post-strutturalismo e del decostruzionismo - torna anche qui il ricorso al barthesiano effetto del reale come strumento privilegiato per risolvere il dilemma posto dal termine "realismo" tramite uno spostamento dell'attenzione verso gli effetti e la sfera estetica delle forme ${ }^{25}$.

Ma se nell'ambito della riflessione sul rapporto tra forma e contenuto le differenze con le aree francese e italiana sembrano segnare una distanza, credo tuttavia che sulla questione del soggetto scrivente, invece, il confronto con l'area tedesca potrebbe rappresentare un importante arricchimento. Non mi riferisco qui tanto a idee nate in seno al dibattito sul nuovo realismo (come la definizione di Ichzeit adottata per l'epoca contemporanea dallo scrittore Maxim Biller $^{26}$ ), quanto piuttosto a un filone di indagine sostanzialmente autonomo, che offre tuttavia possibili collegamenti con il fenomeno del realismo. Si tratta della riflessione sul rapporto tra etica e letteratura: sorta sulla spinta della ethical turn di matrice anglosassone, essa ha assunto una fisionomia autonoma nell'ambito accademico tedesco, sviluppandosi anche attraverso strumenti di diffusione scientifica (come la collana Ethik-Text-Kultur dell'editore Fink, che ospita una ricca serie di volumi di taglio comparatistico e interdisciplinare ${ }^{27}$ ) e strutture universitarie (come il corso di laurea magistrale Ethik der Textkulturen attivato presso le università bavaresi di Augusta e di Erlangen).

In Italia il rapporto tra etica e letteratura, sebbene evocato da voci critiche autorevoli e da esempi letterari di rilievo internazionale ${ }^{28}$, non è tuttavia divenuto oggetto di una riflessione sistematica. In Germania l'indagine ha preso le mosse dalla costatazione che l'epoca postmoderna aveva spazzato via definitivamente l'idea di una possibile subordinazione della letteratura alla morale e che

25. Ibid., p. 241 (ma la categoria di Barthes come "via d'uscita al dilemma" posto dal concetto di realismo è evocata anche nel contributo di apertura del volume di PARR, Rolf, "Neue Realismen. Formen des Realismus in der Gegenwartsliteratur", in part. pp. 16-17).

26. BILLER, Maxim, "Ichzeit. Über die Epoche, in der wir schreiben", Frankfurter Allgemeine Sonntagszeitung, 2 ottobre 2011, p. 23.

27. La collana, che consta attualmente di dodici volumi, è diretta da JACOB, Joachim, Lubkold, Christine, Mayer, Mathias e ÖHlschläger, Claudia.

28. Mi limito a rinviare a uno degli ultimi appelli lanciati da Cesare Segre (cfr. il capitolo "Etica e letteratura", in SEGRE, Cesare, Tempo di bilanci. La Fine del Novecento, Torino, Einaudi, 2005) e agli esempi di Primo Levi e di Roberto Saviano. Il ricorso di quest'ultimo a Levi s'iscrive in una tendenza contemporanea ormai molto diffusa e collegata ai temi del ritorno alla realtà e dell'impegno etico dello scrittore (sull'argomento vedi ora Rondini, Andrea, "Impossibile vivere senza aver letto Se questo è un nomo. La ricezione italiana contemporanea di Primo Levi", in Ticontre. Teoria Testo Traduzione, n. 6, 2016, pp. 1-22). 
occorreva dunque ridefinire tale rapporto. Per questo motivo, l'“etica" viene concepita in opposizione alla "morale" e viene considerata nella sua natura di relazione dialogica, cioè di scambio e di confronto tra il testo e il lettore:

La letteratura contemporanea $[\ldots]$ si è data il compito di mettere a disposizione una nuova consapevolezza dei valori, che vuole diventare oggetto di uno scambio di vedute con il lettore. In tal modo noi, in quanto lettori che agiscono e che si fanno portatori di responsabilità, siamo sollecitati, attraverso il confronto con la letteratura, a prendere posizione all'interno del discorso sociale. Se il movimento postmodernista è stato necessario per potersi affrancare da visioni morali rigidamente normative e per poter rompere vecchie strutture di potere, adesso tuttavia il soggetto viene di nuovo chiamato a un atto di responsabilità, per poter arginare il disorientamento provocato dalla liberazione postmodernista. L'etica narrativa si libera dalla morale normativa nel momento in cui la letteratura viene concepita come forma di scambio di esperienze, come dialogo etico con l'Altro ${ }^{29}$.

Seppure non espressamente menzionato, è qui in gioco il principio dell'interpretazione del testo letterario come dialogo tra testo e lettore, un principio fondamentale della tradizione ermeneutica tedesca, che affonda le sue radici nel pensiero filosofico ottocentesco e che si è poi rinnovato attraverso i maggiori esponenti della critica e della teoria letteraria del Novecento, da Auerbach a Spitzer sino all'estetica della ricezione. Applicarlo al problema etico sollevato dalla letteratura contemporanea significa esplorare le sue potenzialità in una direzione nuova. Già da questo sintetico confronto emerge dunque come un incontro tra le istanze critiche presenti nell'area romanza e quelle germanofone potrebbe rappresentare un'occasione di arricchimento reciproco e mettere a disposizione risorse per osservare gli sviluppi della letteratura contemporanea da nuove angolazioni.

Christian RivoletTI

Friedrich-Alexander-Universität Erlangen-Nürnberg

29. WALDOw, Stephanie, "Mich kümmerts, wer spricht. Literaturwissenschaft heute als ethischer Dialog, in Lubkoll, Christine, WischmeYer, Oda (a cura di), Ethical Turn? Geisteswissenschaften in neuer Verantwortung, München, Fink, 2009, pp. 119-137: 132-133. Della stessa autrice cfr. anche Schreiben als Begegnung mit dem Anderen. Zum Verbältnis von Ethik. und Narration in philosophischen und literarischen Texten der Gegenwart, München, Fink, 2013. 
